\title{
Health-related quality of life and colorectal cancer-specific symptoms in patients with chemotherapy-refractory metastatic disease treated with panitumumab
}

\author{
Dawn Odom • Beth Barber • Lee Bennett • \\ Marc Peeters - Zhongyun Zhao • James Kaye • \\ Michael Wolf • Jeffrey Wiezorek
}

Accepted: 3 December 2010 / Published online: 29 December 2010

(C) The Author(s) 2010. This article is published with open access at Springerlink.com

\begin{abstract}
Purpose Panitumumab monotherapy is approved for chemotherapy-refractory wild-type KRAS metastatic colorectal cancer (mCRC). Patient-reported outcomes - although important in the palliative setting - have not been reported in this patient population.

Methods In a phase 3 trial $(n=463)$, patients with chemotherapy-refractory mCRC were randomized 1:1 to panitumumab plus best supportive care (BSC) or BSC alone. Patient-reported outcomes were assessed using the NCCN/FACT CRC Symptom Index (FCSI) and EQ-5D Index. KRAS tumor status was analyzed in a prospectively defined, retrospective analysis. Average difference in change from baseline between treatment groups was evaluated using linear mixed and pattern-mixture models. Results KRAS tumor status and post-baseline patientreported outcomes were available for 363 patients. Linear mixed models indicated significant differences in the FCSI score (difference in least-squares [LS] adjusted means [95\% $\mathrm{CI}] ; 5.62[2.38,8.86])$ and the EQ-5D Index (difference in
\end{abstract}

D. Odom $\cdot$ L. Bennett $\cdot$ J. Kaye

RTI Health Solutions,

3040 East Cornwallis Road, Post Office Box 12194,

Research Triangle Park, NC 22709-2194, USA

B. Barber $\cdot$ Z. Zhao $(\bowtie) \cdot$ M. Wolf $\cdot$ J. Wiezorek

Amgen Inc,

One Amgen Center Drive,

Thousand Oaks, CA 91320-1799, USA

e-mail: zhongyun@amgen.com

M. Peeters

University Hospital Antwerp,

Wilrijkstraat 10 ,

B-2650 Edegem Antwerp, Belgium
LS adjusted means [95\% CI]; $0.22[0.12,0.32])$ favoring panitumumab over BSC in patients with wild-type KRAS mCRC. By pattern-mixture analysis, the advantage of panitumumab over BSC was more pronounced in those patients with wild-type KRAS mCRC who did not drop out of the study early. In patients with mutant KRAS mCRC, no differences were observed between groups.

Conclusions Panitumumab-treated patients with wild-type KRAS mCRC maintained better control of CRC symptoms and quality of life compared with BSC alone, extending our understanding of the benefits of panitumumab treatment beyond improvements in progression-free survival.

Keywords Panitumumab KRAS . Colorectal cancer . Symptoms $\cdot$ HRQoL

\section{Introduction}

Colorectal cancer (CRC) is a major health concern, representing the third most commonly diagnosed cancer worldwide and the second leading cause of cancer mortality in the developed world [1, 2]. Following significant improvements in the treatment of CRC as a result of advances in systemic chemotherapy [3], use of agents targeting the vascular endothelial growth factor and epidermal growth factor receptor (EGFR) now also have an established role in patients with metastatic disease [4-6].

Panitumumab is a fully human monoclonal antibody directed against the EGFR. Its clinical efficacy in metastatic CRC (mCRC) was confirmed in an open-label, phase 3 trial, in which patients receiving panitumumab plus best supportive care (BSC) had significantly improved 
progression-free survival (PFS) compared with those receiving $\mathrm{BSC}$ alone (hazard ratio [HR] 0.54; 95\% confidence interval $[\mathrm{CI}], 0.44$ to 0.66 ) [6] in patients with chemotherapy-refractory mCRC. Mutations in KRAS-a gene encoding a GTPase that mediates downstream intracellular EGFR signaling - are found in approximately $35-45 \%$ of CRC tumors [7] and have been associated with a lack of response to EGFR inhibitors when used for mCRC [8]. Evaluation of the predictive role of KRAS mutational status in the response to panitumumab in this phase 3 monotherapy study identified that its beneficial effect was restricted to patients whose tumors had wild-type $K R A S$. In patients with wild-type $K R A S \mathrm{mCRC}$, panitumumab plus BSC was associated with a statistically significant improvement in median PFS compared with BSC alone (HR, 0.45; 95\% CI, 0.34-0.59 [9]). In mCRC, the clinical use of panitumumab (like cetuximab) is therefore now restricted to patients with wild-type KRAS tumors. Despite recent advances in the treatment of mCRC provided by targeted agents, most patients will eventually die of the disease. The goal of therapy in this setting is, therefore, to delay disease progression, control symptoms, and maintain health-related quality of life (HRQoL) for as long as possible $[10,11]$. As such, patient-reported outcomes are particularly important to patients with metastatic disease, and the potential for benefit related to these outcomes is a key consideration in the choice of therapy [12].

During the phase 3 trial of panitumumab monotherapy, HRQoL and CRC symptom data were collected. Patientreported outcomes from the trial were briefly reported in the primary efficacy paper but only for the overall patient population and not by KRAS tumor status [6] (online appendix only). Since panitumumab is only indicated for patients with wild-type $K R A S$ tumor status, the available patient-reported outcomes data do not, therefore, reflect the findings for the population for whom treatment with panitumumab is appropriate. In addition, there was a large amount of missing patient-reported outcome data that was unlikely to be missing at random: as expected for patients with advanced refractory disease, more than $50 \%$ of patients in the BSC alone arm and KRAS mutant patients in panitumumab plus BSC arm had progressed by week 8 [6], and thus missing data are likely to have been related to declining health. The last-observation-carried-forward (LOCF) method used in the previous overall population analysis of HRQoL data is therefore a less-than-optimal approach to evaluate the true impact of panitumumab on patient-reported outcomes.

Using data from this phase 3 trial, we therefore sought to evaluate the impact of panitumumab on patient-reported outcomes according to KRAS tumor status and including statistical analyses aimed at properly accounting for the missing data.

\section{Patients and methods}

Patients and study design

The patient population and design for this phase 3, openlabel, randomized, controlled trial $(n=463)$ have been described elsewhere [6,9]. Briefly, patients with EGFRdetectable $\mathrm{mCRC}$ and documented evidence of disease progression after failure of fluoropyrimidines and prespecified exposure to oxaliplatin and irinotecan were randomly assigned to receive panitumumab $6 \mathrm{mg} / \mathrm{kg}$ plus BSC every 2 weeks or BSC alone. The primary endpoint of the study was PFS, with progression assessed by central radiologic review at specified time points from weeks 8 to 48 , then every 3 months thereafter. The KRAS tumor status was evaluated in a prospectively defined, retrospective analysis of formalin-fixed paraffin-embedded tumor sections in a blinded fashion, using a validated KRAS mutation kit [9]. The study protocol was approved by the ethics board at each research center, and patients provided written informed consent, including that for research into the paraffin-embedded tumor samples provided at baseline. An independent clinical research organization (working on behalf of the study sponsor) monitored study centers in Central and Eastern Europe, and representatives of the study sponsor monitored study centers in the rest of world. Monitors were responsible for reviewing adherence to the protocol, compliance with Good Clinical Practice, and the completeness, accuracy, and consistency of the data.

\section{Patient-reported outcome instruments and data collection}

Colorectal cancer symptoms were assessed using the National Comprehensive Cancer Network (NCCN) Functional Assessment of Cancer Therapy, Colorectal Symptom Index (FCSI). The FCSI is a validated, nine-item questionnaire that addresses the most important symptoms associated with CRC, including fatigue, pain, weight loss, diarrhea, nausea, stomach swelling/cramps and appetite, and more general aspects of HRQoL such as ability to enjoy life and contentedness in quality of life (QoL) [13]. Patients responded to each item of this questionnaire using a five-point Likert-type scale ranging from 0 (not at all) to 4 (very much). Raw FCSI scores, which range from 0 to 36 , were converted to total scores ranging from 0 (severely symptomatic in all symptoms assessed) to 100 (symptomfree for all symptoms assessed) using published scoring methodology [13]. The minimal clinically important difference (MCID) was defined as a change in score of 3 points or more [14]. This instrument was administered at baseline and then every 2 weeks until disease progression.

Overall HRQoL was measured at baseline and monthly until disease progression using the EuroQol 5-Dimensions 
(EQ-5D) Index. The EQ-5D Index is a generic preferencebased measure of overall QoL, as assessed across five dimensions: mobility, self-care, usual activity, pain or discomfort, and anxiety or depression. Each dimension has three possible responses (no problems, moderate problems, or extreme problems). The EQ-5D Index score is calculated by combining scores from the five dimensions using population-based preference weights and ranges from -0.594 to 1 [15]. The MCID for the EQ-5D Index in patients with $\mathrm{mCRC}$ has been estimated as a change in score of 0.08 points or more [16].

\section{Statistical analysis}

The primary objective of this analysis was to estimate the average difference in effect of panitumumab plus BSC treatment compared with BSC alone on the FCSI and EQ-5D Index, both overall and for the wild-type and mutant KRAS subgroups. KRAS tumor status was ascertained in $92 \%$ of patients enrolled in the phase 3 trial [9]. The analysis set was defined as all patients in the intent-totreat population (all subjects who gave consent and were randomized) who had at least one post-baseline FCSI or EQ-5D Index assessment and an assessed KRAS status ("KRAS patient-reported outcome (PRO) analysis set"). Only outcomes obtained through week 17 of the study were used due to small sample sizes after week 17. Change in score from baseline was analyzed over time using linear mixed models for repeated measures [17]. The models included explanatory variables for study treatment arm, study week, and the interaction between treatment arm and study week. Each repeated measures model also included adjustments for baseline outcome score, baseline collection medium, baseline Eastern Cooperative Oncology Group (ECOG) performance status, and geographic region. Treatment-specific estimates of the average change in each outcome score from baseline, along with 95\% CI, were calculated for the overall cohort and for each KRAS subgroup using least-squares mean (LSM) differences.

To evaluate the effect of study attrition on the estimates of treatment differences, a sensitivity analysis was performed using pattern-mixture models [18, 19], which are linear models that incorporate information about missing data. For each endpoint, patients were first categorized according to the last week for which outcome data were collected up to week 17. These categories were then collapsed into larger groups of patients representing two general patterns of missing response data: those who dropped out of the study early ("early dropout") and those who either dropped out later or completed all assessments up to week 17 ("late dropout/ completer"). For the EQ-5D Index, we defined the "early dropout" group as those who dropped out on or before week 9 and the "late dropout/completer" group as those who dropped out after week 9 or did not drop out of the study early. To account for the more frequent assessments, FCSI "early dropout" group was defined as those who dropped out before week 9 and the "late dropout/completer" group as those who dropped out on or after week 9 or did not drop out.

Dropout status was incorporated into pattern-mixture models of change in score from baseline for each outcome. These models included fixed effects for treatment arm, study week, dropout pattern group, and interactions between these effects. The model included random effects to account for variability in outcome scores at baseline and over time among patients. Within each dropout group, treatment-specific LSM estimates of average change in each outcome score from baseline, along with $95 \%$ CI, were calculated for each $K R A S$ subgroup and overall. All statistical analyses were performed using SAS for Linux, Version 9.1 (SAS Institute).

\section{Results}

Of the 463 patients randomly assigned to treatment in the main study, information on tumor KRAS status together with post-baseline patient-reported outcome data was available for 363 patients (KRAS PRO analysis set, $n=188$ panitumumab plus BSC; $n=175$ BSC alone), representing $78 \%$ of the overall study population. Baseline characteristics were generally well-balanced across treatment groups and were similar to those reported in the overall patient population [6], with the exception of a slightly higher percentage of better performing patients $(E C O G=0)$ in the panitumumab plus BSC compared with the BSC alone treatment group, overall and for each KRAS group (Table 1).

\section{Patient-reported CRC symptoms and HRQoL}

Table 2 presents the number of patients completing FCSI questionnaires for each week until week 17. In line with previous reports $[20,21]$, the amount of missing data was substantial, particularly in the later weeks, although patients with wild-type $K R A S$ mCRC receiving panitumumab plus BSC had a higher percentage of available data for each post-baseline week compared with those receiving BSC alone. Similar results were seen for the EQ-5D Index (data not shown).

\section{Change in scores from baseline-linear mixed model}

According to the repeated-measures model of change from baseline score, there was less deterioration in the FCSI score and EQ-5D Index in the panitumumab plus BSC group compared with the BSC alone group, both overall and for the subgroup of patients with wild-type $K R A S$ 
Table 1 Baseline characteristics of randomized patients with information on tumor KRAS status plus post-baseline data on FCSI score and EQ-5D Index (KRAS PRO analysis set)

Panitumumab plus BSC

\begin{tabular}{lll}
\hline $\begin{array}{l}\text { Overall } \\
(n=188), n(\%)\end{array}$ & $\begin{array}{l}\text { Wild-type KRAS } \\
(n=112), n(\%)\end{array}$ & $\begin{array}{l}\text { Mutant KRAS } \\
(n=76), n(\%)\end{array}$
\end{tabular}

BSC alone

$\begin{array}{lll}\text { Overall } & \text { Wild-type KRAS } & \text { Mutant KRAS } \\ (n=175), n(\%) & (n=96), n(\%) & (n=79), n(\%)\end{array}$

\begin{tabular}{|c|c|c|c|c|c|c|}
\hline \multicolumn{7}{|l|}{ Sex } \\
\hline Men & $123(65)$ & $79(701)$ & $44(58)$ & $113(65)$ & $62(645)$ & $51(65)$ \\
\hline Women & $65(35)$ & $33(29)$ & $32(42)$ & $62(35)$ & $34(35)$ & $28(35)$ \\
\hline \multicolumn{7}{|l|}{ Race/ethnicity } \\
\hline White & $187(99)$ & $111(99)$ & $76(100)$ & $171(98)$ & $95(99)$ & $76(96)$ \\
\hline Other & $1(1)$ & $1(1)$ & $0(0)$ & $4(2)$ & $1(1)$ & $3(4)$ \\
\hline \multicolumn{7}{|l|}{ Age, years } \\
\hline Mean (SD) & $61(10)$ & $62(10)$ & $60(11)$ & $62(10)$ & $62(10)$ & $61(11)$ \\
\hline \multicolumn{7}{|c|}{ Primary diagnosis } \\
\hline Colon cancer & $126(67)$ & $78(70)$ & $48(63)$ & $117(67)$ & $68(71)$ & $49(62)$ \\
\hline Rectal cancer & $62(33)$ & $34(30)$ & $28(37)$ & $58(33)$ & $28(29)$ & $30(38)$ \\
\hline \multicolumn{7}{|l|}{ ECOG } \\
\hline 0 & $91(48)$ & $52(46)$ & $39(51)$ & $62(35)$ & $35(36)$ & $27(34)$ \\
\hline 1 & $76(40)$ & $50(45)$ & $26(34)$ & $91(52)$ & $51(53)$ & $40(51)$ \\
\hline 2 & $21(11)$ & $10(9)$ & $11(14)$ & $22(13)$ & $10(10)$ & $12(15)$ \\
\hline \multicolumn{7}{|c|}{ Time since primary diagnosis, months } \\
\hline Mean (SD) & $31(22)$ & $33(25)$ & $27(17)$ & $32(21)$ & $31(20)$ & $34(21)$ \\
\hline \multicolumn{7}{|c|}{ Time since metastatic disease, months } \\
\hline Mean (SD) & $21(10)$ & $22(10)$ & $20(10)$ & $22(11)$ & $24(13)$ & $19(8)$ \\
\hline \multicolumn{7}{|c|}{ Baseline EQ-5D Index } \\
\hline Mean (SD) & $0.72(0.24)$ & $0.73(0.24)$ & $0.71(0.25)$ & $0.68(0.25)$ & $0.68(0.23)$ & $0.68(0.26)$ \\
\hline \multicolumn{7}{|c|}{ Baseline FSCI score } \\
\hline Mean (SD) & $72.27(13.69)$ & $73.21(13.05)$ & $70.94(14.55)$ & $71.84(14.28)$ & $71.78(13.48)$ & $71.91(15.28)$ \\
\hline
\end{tabular}

FCSI Functional Assessment of Cancer Therapy, Colorectal Symptom Index, EQ-5D Index EuroQol 5-Dimensions Index, PRO patient-reported outcomes, $B S C$ best supportive care, $S D$ standard deviation

mCRC. For the FCSI scores, an average LSM difference between treatment groups across all weeks favored panitumumab by $3.60(95 \% \mathrm{CI}, 0.90$ to 6.30$)$ overall and 5.62 (95\% CI, 2.38 to 8.86) for patients with wild-type $K R A S$
mCRC (Fig. 1a). The results of EQ-5D Index also favored panitumumab, with an average LSM difference between treatment groups across all weeks of 0.17 (95\% CI, 0.09 to 0.25 ) overall and 0.22 (95\% CI, 0.12 to 0.32 ) for patients

Table 2 Number of patients completing the FCSI (KRAS PRO analysis set)

\begin{tabular}{|c|c|c|c|c|c|c|}
\hline & \multicolumn{3}{|c|}{ Panitumumab plus BSC } & \multicolumn{3}{|c|}{$\mathrm{BSC}$ alone } \\
\hline & Overall & Wild-type $K R A S$ & Mutant $K R A S$ & Overall & Wild-type $K R A S$ & Mutant $K R A S$ \\
\hline Number of patients & 188 & 112 & 76 & 175 & 96 & 79 \\
\hline Week 1 (baseline) & 177 & 104 & 73 & 172 & 94 & 78 \\
\hline Week 3 & 167 & 102 & 65 & 139 & 78 & 61 \\
\hline Week 5 & 170 & 103 & 67 & 123 & 78 & 45 \\
\hline Week 7 & 152 & 96 & 56 & 91 & 62 & 29 \\
\hline Week 9 & 100 & 70 & 30 & 47 & 28 & 19 \\
\hline Week 13 & 80 & 63 & 17 & 13 & 9 & 4 \\
\hline Week 17 & 62 & 56 & 6 & 7 & 6 & 1 \\
\hline
\end{tabular}

FCSI Functional Assessment of Cancer Therapy, Colorectal Symptom Index, PRO patient-reported outcomes, BSC best supportive care 

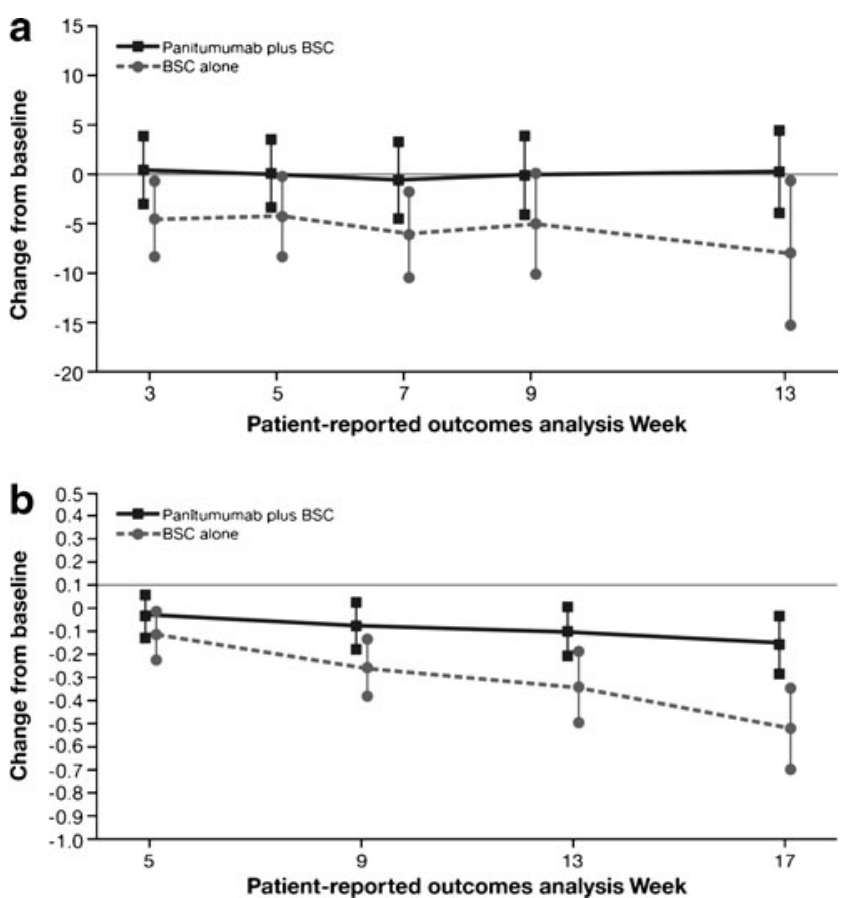

Fig. 1 a Least-squares mean differences in the change from baseline in the FCSI scores between panitumumab plus BSC and BSC alone for patients with wild-type $K R A S \mathrm{mCRC}$ by analysis week, linear mixed models. Note: This model includes data only through week 13. Inclusion of week 17 data, which are missing for more than $80 \%$ of the analysis population, caused model instability to the extent that treatment effects could not be estimated. b Least-squares mean differences in the change from baseline in the EQ-5D Index between panitumumab plus BSC and BSC alone for patients with wild-type $K R A S$ mCRC by analysis week (KRAS PRO analysis set), linear mixed models. FSCI Functional Assessment of Cancer Therapy, Colorectal Symptom Index; $B S C$ best supportive care; $m C R C$ metastatic colorectal cancer; EQ-5D EuroQol 5-Dimensions Index

with wild-type $K R A S$ mCRC (Fig. 1b). Among patients with wild-type $K R A S \mathrm{mCRC}$, in particular, the differences between treatment groups substantially exceeded each instrument's MCID. Most notably, the difference between treatments in EQ-5D Index change was 2.75 times higher than the MCID in patients with wild-type KRAS mCRC. As expected, analysis of the mutant $K R A S$ group did not show any significant differences in change from baseline FCSI score or EQ-5D Index between treatments (FCSI score: average LSM difference between treatment groups across all weeks of -1.41 [95\% CI, -6.41 to 3.59]; EQ-5D Index: average LSM difference between treatment groups across all weeks of 0.08 [95\% CI, -0.04 to 0.19$]$ ).

\section{Pattern-mixture model (sensitivity analysis)}

For each outcome, missing data were categorized into early and late dropout patterns, regardless of treatment group or $K R A S$ status. Based on a simple cross-sectional average, Fig. 2 illustrates that there was a clear difference in the

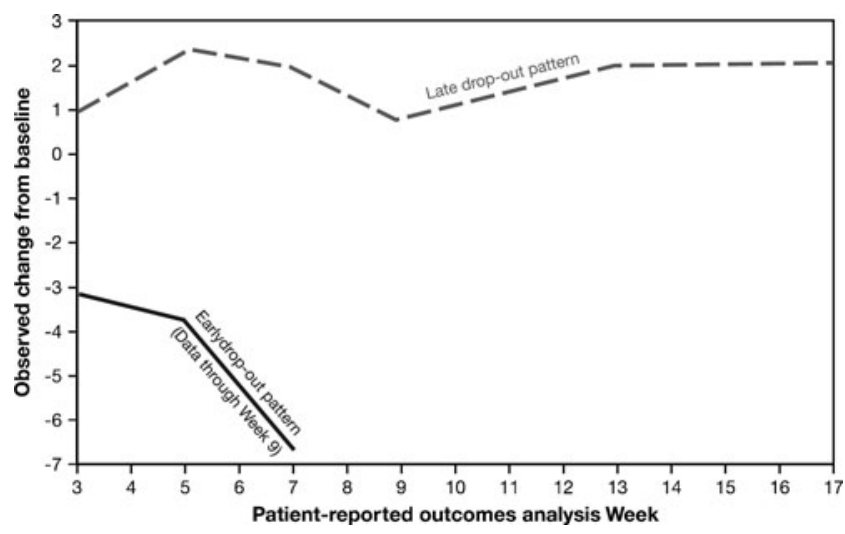

Fig. 2 Unadjusted average change from baseline in FCSI score by week of assessment and dropout pattern (KRAS PRO analysis set). FSCI Functional Assessment of Cancer Therapy, Colorectal Symptom Index

FCSI score of the early and late dropout groups at each time point. Similar results were seen for the EQ-5D Index (data not shown).

For both the FCSI score and EQ-5D Index, the panitumumab plus BSC group had a significantly higher proportion of patients in the late dropout group than in the early dropout group, whereas the BSC alone group had a significantly higher proportion of patients in the early dropout group $(P<0.001$; Table 3$)$. Together, these data indicate that the dropout pattern was informative about the treatment group as well as the patient-reported outcomes, and thus the missing data were considered to be non-random, and treatment effects were evaluated separately for those patients who did and those who did not drop out early.

Table 4 displays the results of the pattern-mixture analyses modeling average differences in change from baseline score for the FCSI and EQ-5D Index. For late dropout patients with wild-type KRAS mCRC, those receiving panitumumab experienced an initial increase in EQ-5D Index and FCSI scores that was not observed in patients receiving $\mathrm{BSC}$ alone. In this group, the LSM difference between groups was statistically significant in favor of panitumumab with differences in both instruments exceeding the relevant MCIDs, consistent with the findings from the repeated-measures model (Table 4). On the EQ-5D index, differences between treatment groups were four times higher than the MCID and substantially exceeded the panitumumab advantage seen in the repeated-measures model. These treatment advantages were not evident for patients in the early dropout pattern group with wild-type KRAS mCRC (Table 4). Similar to the linear model, there were also no significant differences between the treatments in patients with mutant KRAS mCRC (Table 4).

Based on the FCSI assessment of symptoms among patients with wild-type KRAS mCRC who stayed on study, 
Table 3 Summary of dropout pattern by treatment (KRAS PRO analysis set)

\begin{tabular}{lcc}
\hline Instrument/pattern & Panitumumab plus BSC $(n=188), n(\%)$ & BSC $(n=175), n(\%)$ \\
\hline FCSI & & $P$ value \\
Early dropout & $75(42)$ & $109(68)$ \\
Late dropout/completer & $101(57)$ & $49(31)$ \\
EQ-5D Index & & $99(68)$ \\
Early dropout & $65(38)$ & $47(32)$ \\
Late dropout/completer & $105(62)$ & $<0.001$ \\
\hline
\end{tabular}

Patients with missing data for all post-baseline assessments were excluded from the analysis. $P$ values are based on the Pearson chi-square test $P R O$ patient-reported outcomes, BSC best supportive care, FCSI Functional Assessment of Cancer Therapy, Colorectal Symptom Index, $E Q-5 D$ Index EuroQol 5-Dimensions Index

the treatment advantage favoring panitumumab appeared to be consistent across all weeks of the study (Fig. 3a), whereas the treatment advantage favoring panitumumab on the EQ-5D Index became larger as time increased, with the greatest advantage over BSC alone seen at week 17 (Fig. 3b).

\section{Discussion}

Patient-reported outcomes such as cancer-related symptomatology and QoL are increasingly being recognized as important aspects of palliative care in the advanced cancer setting, providing important information to help make treatment decisions [12]. In this analysis, patients treated with panitumumab showed significantly better control of CRC symptoms and maintenance of HRQoL compared with those who received BSC alone: this benefit was most evident among the subgroup of patients with wild-type $K R A S$ mCRC and was not seen in patients with mutant $K R A S$ mCRC, or in those dropping out of the study early. This therefore illustrates, for the first time, the beneficial effects of panitumumab on HRQoL in patients with wildtype $K R A S \mathrm{mCRC}$, the population for whom panitumumab treatment is indicated in clinical practice. Indeed, in those patients with wild-type $K R A S$ mCRC treated with panitumumab who did not drop out of the study early, a decrease in CRC-related symptoms and an increase in HRQoL was observed by week 3 of the study. This is consistent with the finding that the beneficial effects of panitumumab on PFS are limited to patients with wild-type KRAS mCRC [9] and with previous analyses indicating that a lack of disease progression at week 8 provided by panitumumab was associated with better HRQoL and symptom control [20]. For HRQoL and CRC symptoms, the benefits of panitumumab compared with BSC alone in patients with wildtype KRAS mCRC exceeded the established MCID levels, indicating that statistical differences were also clinically relevant. To our knowledge, our analyses are the first to illustrate the beneficial effects of an EGFR inhibitor on CRC-specific symptomatology, which is particularly important given that treatment of patients with mCRC is primarily given with palliative intent $[10,11]$.

The HRQoL of patients with advanced CRC treated with the EGFR inhibitor cetuximab, overall and by KRAS status, has also been published recently [22]. The findings were generally consistent with ours in that HRQoL benefits of cetuximab were observed and were most apparent in the subgroup of patients with wild-type KRAS mCRC. However, the results cannot be more closely compared, as the

Table 4 Least-squares mean differences $(95 \% \mathrm{CI})$ in the change from baseline in the EQ-5D Index score and FCSI scores between panitumumab plus BSC vs BSC alone: overall and by tumor KRAS status, using pattern-mixture models (KRAS PRO analysis set)

\begin{tabular}{|c|c|c|c|c|c|c|}
\hline \multirow[b]{2}{*}{ Dropout pattern } & \multicolumn{3}{|l|}{ EQ-5D Index } & \multicolumn{3}{|l|}{ FCSI } \\
\hline & Overall & Wild-type $K R A S$ & Mutant $K R A S$ & Overall & Wild-type $K R A S$ & Mutant $K R A S$ \\
\hline Early dropout & $-0.08(-0.21,0.05)$ & $-0.19(-0.38,0.01)$ & $-0.02(-0.19,0.15)$ & $0.53(-3.15,4.20)$ & $-2.21(-7.16,2.75)$ & $4.27(-1.33,9.88)$ \\
\hline $\begin{array}{l}\text { Late dropout/ } \\
\text { completer }\end{array}$ & $0.26^{\mathrm{a}, *}(0.16,0.37)$ & $0.32^{\mathrm{a}, *}(0.18,0.45)$ & $0.13(-0.03,0.29)$ & $3.63(-0.05,7.31)$ & $5.75^{*, \mathrm{a}}(1.45,10.04)$ & $-0.66(-7.27,5.95)$ \\
\hline
\end{tabular}

CI, confidence interval EQ-5D Index EuroQol 5-Dimensions Index; FCSI Functional Assessment of Cancer Therapy, Colorectal Symptom Index; $B S C$ best supportive care; $P R O$ patient-reported outcomes

${ }^{a}$ Exceeds minimum clinically important difference (EQ-5D Index $=0.08$ points [16]; FCSI=3 points [14])

$* P \leq 0.05$ 


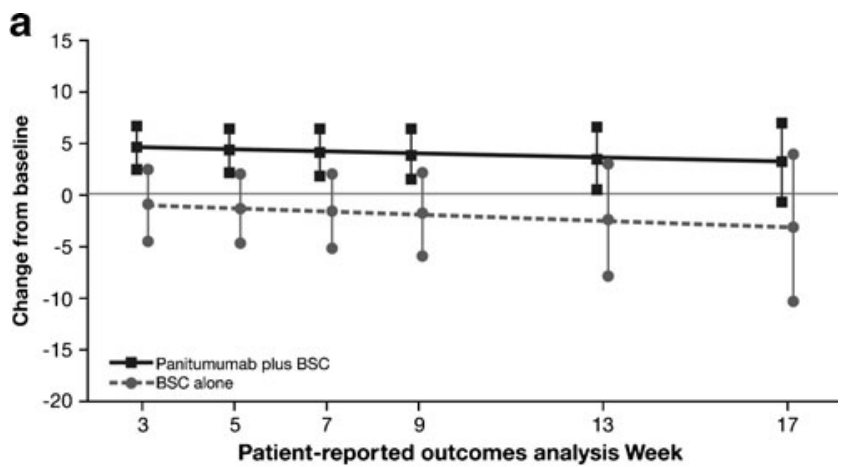

b

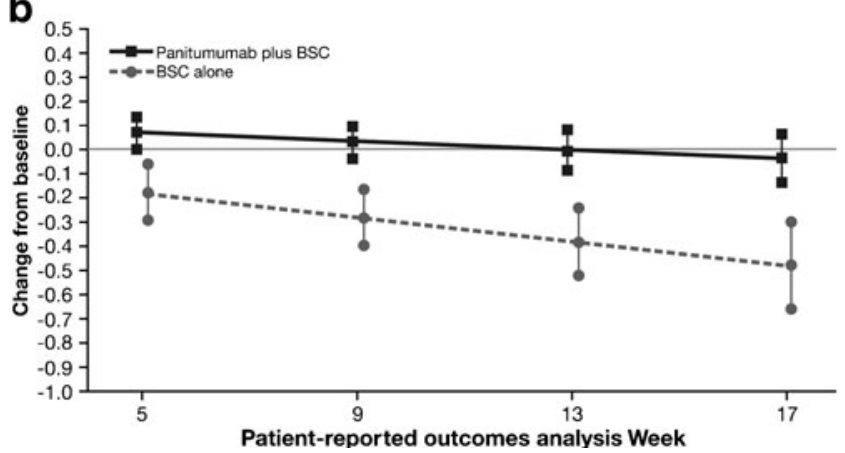

Fig. 3 a Least-squares mean differences in the change from baseline in the FCSI scores between panitumumab plus BSC and BSC alone for patients with wild-type $K R A S \mathrm{mCRC}$ in the late dropout group by analysis week, pattern-mixture model. b Least-squares mean differences in the change from baseline in the EQ-5D Index score between panitumumab plus BSC and BSC alone for patients with wild-type $K R A S$ mCRC in the late dropout group by analysis week, patternmixture model. FSCI Functional Assessment of Cancer Therapy, Colorectal Symptom Index, BSC best supportive care, $m C R C$ metastatic colorectal cancer, EQ-5D EuroQol 5-Dimensions Index

cetuximab analysis used data from the EORTC QLQ-C30, which includes cancer-related (but not CRC-specific) symptoms [22], whereas we chose to evaluate HRQoL and $\mathrm{CRC}$-specific symptoms separately. In addition, in the study of $\mathrm{Au}$ et al. [22], HRQoL data continued to be collected after disease progression. Inclusion of such data was not possible in our analysis since the study was designed to allow cross-over from BSC to panitumumab at this point [6].

Taken together with those of the cetuximab analyses [22], these HRQoL results may, however, contribute to a more accurate evaluation of the balance between the positive effects of EGFR inhibitors and the side effects associated with their use, such as skin toxicity, which are generally perceived to have a detrimental effect on HRQoL [23]. In the main study population of the phase 3 trial, skin toxicity occurred in $90 \%$ of patients in the panitumumab group compared with $9 \%$ of patients in the BSC group [6] and was more likely to develop in patients remaining on the study because of treatment benefit [21]. Despite the association of skin toxicity with prolonged treatment, the benefits of panitumumab in terms of HRQoL and symptomatology were most apparent in the group of patients with wild-type KRAS mCRC who did not drop out of the study early in our analysis. Indeed, more severe skin toxicity in association with panitumumab has been associated with higher rather than lower HRQoL scores [21]. Similar findings in relation to skin toxicity and HRQoL have also been observed with cetuximab and have been suggested to reflect an understanding by patients that skin rash is indicative of a response to therapy, which helps them cope with this side effect [24]. As noted by Au et al. [22], the presence of skin rash also introduces the potential for bias in the HRQoL results, since those patients experiencing it may have been more likely to report improvements if they were aware that the rash was a known predictor of benefit. However, this suggestion would be at odds with the fact that the benefits of panitumumab in terms of HRQoL were most prominent in patients with wild-type KRAS $\mathrm{mCRC}$ who were more likely to experience objective tumor control than those with KRAS mutant tumors, and these HRQoL benefits were not seen in patients with mutant $K R A S$ mCRC despite the fact that the vast majority of this subpopulation also developed skin rash.

Our results differ from the original protocol-specified analysis of HRQoL and CRC symptom control, which did not take into account KRAS mutation status and which used the LOCF imputation method. This showed a numerical trend favoring panitumumab that did not reach statistical significance [6]. The differences in outcome highlight both the importance of tumor KRAS status and the need to properly account for missing HRQoL data in trials of latestage disease in which the reasons for missing observations are often related to deterioration in a patient's clinical status and so, cannot be classified as missing at random [25]. For a patient who has rapid disease progression and drops out of the study early, LOCF analysis is likely to impute values indicating better health than the true value, potentially biasing the comparison in favor of less effective or less well-tolerated treatment. An advantage to using the repeated-measures models (as in our analysis) is that imputation is not required. Pattern-mixture models provide even greater flexibility since the methodology provides a way to incorporate information about patient dropout into the longitudinal model.

Our sensitivity analyses showed that there were significantly fewer early dropouts for both the HRQoL and CRC symptom scales among the group receiving panitumumab than among the group receiving BSC alone. This disproportionate percentage of data by treatment group likely resulted from the higher rate of discontinuation due to death or disease progression in the BSC only treatment arm [6], and it was important to account for these non-random 
missing data. Of note is that similar findings regarding disproportionate missing data were observed in the cetuximab analysis of Au et al. [22] (although not to the same extent as there was an attempt to collect HRQoL data beyond disease progression), and similar approaches to account for missing data were included in their sensitivity analysis [22].

There are several possible explanations for the apparent lack of effect of panitumumab, in those patients (even with wild-type KRAS mCRC) who dropped out of the study early. Patients treated with panitumumab plus BSC who left the study early due to tolerability concerns or other reasons unrelated to disease progression may not have received enough treatment to yield an improvement in HRQoL or CRC symptoms. Alternatively, the treatment may not have been effective among a subgroup of patients, leading to relatively rapid progression of disease and early dropout.

A potential limitation of this study is that KRAS mutation status was determined retrospectively and therefore was not a stratification factor in the randomization. However, 92\% of patients randomized had KRAS status available, and patients with KRAS mutations were well distributed between the two treatment groups. Although the post hoc nature of our exploratory analysis by KRAS status is a theoretical limitation, the comparison of baseline demographics suggests that, within the KRAS subgroups, patient characteristics were similar between the two treatment arms. Another potential limitation is the imperfect sensitivity and specificity of patient-reported outcome measures. However, our analysis used validated instruments, and our findings are supported by their consistency with the results of the primary study endpoint, PFS.

In conclusion, our analyses illustrate the clinically significant beneficial effects of panitumumab in terms of CRCspecific symptom control and maintenance of HRQoL in patients with wild-type KRAS mCRC, and these findings extend our understanding of the benefits of panitumumab treatment beyond improvements in PFS. These benefits were apparent despite skin toxicity associated with panitumumab treatment and provide important additional information for both patients and physicians when making treatment decisions for mCRC, particularly when the treatment intent is palliative.

\begin{abstract}
Acknowledgments The authors gratefully acknowledge the patients who participated in this trial and their families. All authors had full access to the data and had final responsibility for the decision to submit the manuscript. The study was funded by Amgen Inc. Medical writing assistance was provided by Dr Sue Laing, ScopeMedical Ltd, funded by Amgen Inc.
\end{abstract}

Conflicts of interest Beth Barber, Zhongyun Zhao, Michael Wolf, and Jeffrey Wiezorek are employees and stockholders of Amgen Inc. Marc Peeters acted as an Investigator in the trial discussed in this manuscript and has served as a speaker, a consultant, and an advisory board member for Amgen, Lilly, Merck, Novartis, Roche, and SanofiAventis. Dawn Odom, Lee Bennett, and James Kaye are employees of
RTI Health Solutions and received funding from Amgen Inc to perform the analyses reported in this manuscript.

Open Access This article is distributed under the terms of the Creative Commons Attribution Noncommercial License which permits any noncommercial use, distribution, and reproduction in any medium, provided the original author(s) and source are credited.

\section{References}

1. Parkin DM, Bray F, Ferlay J, Pisani P (2005) Global cancer statistics 2002. CA Cancer J Clin 55:74-108

2. Jemal A, Siegel R, Ward E, Hao Y, Xu J, Murray T, Thun MJ (2008) Cancer statistics, 2008. CA Cancer J Clin 58:71-96

3. Davies JM, Goldberg RM (2008) First-line therapeutic strategies in metastatic colorectal cancer. Oncology (Williston Park) 22:1470-1479

4. Hurwitz H, Fehrenbacher L, Novotny W, Cartwright T, Hainsworth J, Heim W, Berlin J, Baron A, Griffing S, Holmgren E, Ferrara N, Fyfe G, Rogers B, Ross R, Kabbinavar F (2004) Bevacizumab plus irinotecan, fluorouracil, and leucovorin for metastatic colorectal cancer. N Engl J Med 350:2335-2342

5. Jonker DJ, O'Callaghan CJ, Karapetis CS, Zalcberg JR, Tu D, Au HJ, Berry SR, Krahn M, Price T, Simes RJ, Tebbutt NC, van Hazel G, Wierzbicki R, Langer C, Moore MJ (2007) Cetuximab for the treatment of colorectal cancer. N Engl J Med 357:2040-2048

6. Van Cutsem E, Peeters M, Siena S, Humblet A, Neyns B, Canon JL, Van Laethem JL, Maurel J, Richardson G, Wolf M, Amado RG (2007) Open-label phase III trial of panitumumab plus best supportive care compared with best supportive care alone in patients with chemotherapy-refractory metastatic colorectal cancer. J Clin Oncol 25:1658-1664

7. Siena S, Sartore-Bianchi A, Di Nicolantonio F, Balfour J, Bardelli A (2009) Biomarkers predicting clinical outcome of epidermal growth factor receptor-targeted therapy in metastatic colorectal cancer. J Natl Cancer Inst 101:1308-1324

8. Karapetis CS, Khambata-Ford S, Jonker DJ, O'Callaghan CJ, Tu D, Tebbutt NC, Simes RJ, Chalchal H, Shapiro JD, Robitaille S, Price TJ, Shepherd L, Au HJ, Langer C, Moore MJ, Zalcberg JR (2008) K-ras mutations and benefit from cetuximab in advanced colorectal cancer. N Engl J Med 359:1757-1765

9. Amado RG, Wolf M, Peeters M, Van Cutsem E, Siena S, Freeman DJ, Juan T, Sikorski R, Suggs S, Radinsky R, Patterson SD, Chang DD (2008) Wild-type $K R A S$ is required for panitumumab efficacy in patients with metastatic colorectal cancer. J Clin Oncol 26:1626-1634

10. Wiseman LR, Lyseng-Williamson KA (2005) Management of metastatic colorectal cancer: defining the role of capecitabine. Dis Manage Health Outcomes 13:137-149

11. Tappenden P, Jones, R, Paisley S, Carroll C (2007) Systematic review and economic evaluation of bevacizumab and cetuximab for the treatment of metastatic colorectal cancer. Health Technology Assessment 11:No. 12

12. Byrne C, Griffin A, Blazeby J, Conroy T, Efficace F (2007) Healthrelated quality of life as a valid outcome in the treatment of advanced colorectal cancer. Eur J Surg Oncol 33(Suppl 2):S95-S104

13. Cella D, Paul D, Yount S, Winn R, Chang CH, Banik D, Weeks J (2003) What are the most important symptom targets when treating advanced cancer? A survey of providers in the National Comprehensive Cancer Network (NCCN). Cancer Invest 21:526-535

14. Colwell HH, Mathias SD, Turner MP, Lu J, Wright N, Peeters M, Cella D, Devercelli G (2010) Psychometric evaluation of the FACT Colorectal Cancer Symptom Index (FCSI-9): reliability, validity, responsiveness, and clinical meaningfulness. Oncologist 15:308-316 
15. Shaw JW, Johnson JA, Coons SJ (2005) US valuation of the EQ5D health states: development and testing of the D1 valuation model. Med Care 43:203-220

16. Mathias SD, Pritchard ML, Colwell HH, Lu J, Wright N (2006) What is the minimal clinically important difference and responsiveness of a patient-reported outcome questionnaire for metastatic colorectal cancer? Ann Oncol 17: Abstract no. 349P

17. Fitzmaurice GM, Laird N, Ware JH (2004) Applied longitudinal analysis. Wiley, Hoboken

18. Hedeker D, Gibbons RD (1997) Application of random-effects pattern-mixture models for missing data in longitudinal studies. Psychol Methods 2:64

19. Little RJA (1993) Pattern-mixture models for multivariate incomplete data. J Am Stat Assoc 88:125

20. Siena S, Peeters M, Van Cutsem E, Humblet Y, Conte P, Bajetta E, Comandini D, Bodoky G, Van Hazel G, Salek T, Wolf M, Devercelli G, Woolley M, Amado RG (2007) Association of progression-free survival with patient-reported outcomes and survival: results from a randomised phase 3 trial of panitumumab. Br J Cancer 97:1469-1474

21. Peeters M, Siena S, Van Cutsem E, Sobrero A, Hendlisz A, Cascinu S, Kalofonos H, Devercelli G, Wolf M, Amado RG
(2009) Association of progression-free survival, overall survival, and patient-reported outcomes by skin toxicity and $K R A S$ status in patients receiving panitumumab monotherapy. Cancer 115:15441554

22. Au H-J, Karapetis CS, O'Callaghan CJ, Tu D, Moore MJ, Zalcberg JR, Kennecke H, Shapiro JD, Koski S, Pavlakis N, Charpentier D, Wyld D, Jefford M, Knight GJ, Magoski NM, Brundage MD, Jonker DJ (2009) Health-related quality of life in patients with advanced colorectal cancer treated with cetuximab: overall and KRAS-specific results of the NCIC CTG and AGITG CO.17 Trial. J Clin Oncol 11:1822-1828

23. Lynch TJ Jr, Kim ES, Eaby B, Garey J, West DP, Lacouture ME (2007) Epidermal growth factor receptor inhibitor-associated cutaneous toxicities: an evolving paradigm in clinical management. Oncologist 12:610-621

24. Romito F, Giuliani F, Cormio C, Tulipani C, Mattioli V, Colucci G (2010) Psychological effects of cetuximab-induced cutaneous rash in advanced colorectal cancer patients. Support Care Cancer 18:329-334

25. Donaldson GW, Moinpour CM (2005) Learning to live with missing quality-of-life data in advanced-stage disease trials. J Clin Oncol 23:7380-7384 